\title{
RF BREAKDOWN IN NORMAL CONDUCTING SINGLE-CELL STRUCTURES *
}

\author{
V.A. Dolgashev, S.G. Tantawi, C.D. Nantista, SLAC, Menlo Park, CA, 94025, USA \\ Y. Higashi, T. Higo, KEK, Tsukuba, Ibaraki 305, Japan
}

\begin{abstract}
Operating accelerating gradient in normal conducting accelerating structures is often limited by rf breakdown. The limit depends on multiple parameters, including input rf power, rf circuit, cavity shape and material. Experimental and theoretical study of the effects of these parameters on the breakdown limit in full scale structures is difficult and costly. We use $11.4 \mathrm{GHz}$ single-cell traveling wave and standing wave accelerating structures for experiments and modeling of rf breakdown behavior. These test structures are designed so that the electromagnetic fields in one cell mimic the fields in prototype multicell structures for the $\mathrm{X}$-band linear collider. Fields elsewhere in the test structures are significantly lower than that of the single cell. The setup uses matched mode converters that launch the circular $\mathrm{TM}_{01}$ mode into short test structures. The test structures are connected to the mode launchers with vacuum of flanges. This setup allows economic testing of different cell geometries, cell materials and preparation techniques with short turn-around time. Simple 2D geometry of the test structures simplifies modeling of the breakdown currents and their thermal effects.
\end{abstract}

\section{INTRODUCTION}

Accelerating gradient is one of the crucial parameters affecting the design, construction and cost of next-generation linear accelerators. The Next Linear Collider (NLC) / Global Linear Collider (GLC) designs specify unloaded accelerating gradient of $65 \mathrm{MV} / \mathrm{m}$ at $11.4 \mathrm{GHz}$. The CERN based CLIC design requires $150 \mathrm{MV} / \mathrm{m}$ loaded gradient [1]. The major obstacle to higher gradient is rf breakdown.

RF breakdown limits working power and produces irreversible surface damage in high power rf components and rf sources. For a given $\mathrm{rf}$ frequency, the maximum working gradient depends on the rf circuit, structure geometry and material. It is also a function of input power, pulse width, and surface electric and magnetic fields. Here we define working gradient as a gradient with very low breakdown rate - less then one breakdown in $2 \times 10^{6} \mathrm{rf}$ pulses.

The complexity of rf breakdown phenomena and the absence of a proven theory for it make it difficult to apply experimental results on breakdown limit from one rf structure to another. To date, dozens of full-scale travelling wave (TW) structures [2, 3, 4], standing wave (SW) structures [5], and high power waveguides [6] have been tested at SLAC in an effort to produce accelerating structures that satisfy NLC/GLC requirements on gradient and breakdown rate. Although the NLC specified gradient has been

\footnotetext{
* This work was supported by the U.S. Department of Energy contract
} DE-AC02-76SF00515. achieved, many questions about the physics of rf breakdown remain unanswered. The experiments described in this paper are designed to study the breakdown phenomena. They allow economical testing of structures with different cell geometries, materials and preparation techniques with short turn around time.

\section{RF BREAKDOWN}

Below we briefly summarize properties of rf breakdown phenomena obtained during NLC/GLC development.

\section{General Properties of a Breakdown Event}

$\mathrm{RF}$ breakdown is a phenomenon that abruptly and significantly changes transmission and reflection of the rf power directed toward the structure under test. Breakdown is accompanied by a burst of x-rays and by a bright flash of visible light. Main properties of the rf breakdown in TW structures and waveguides [7] are: during breakdown the transmitted power drops to unmeasurable levels with a time constant of 20-200 ns; up to $80 \%$ of the incident rf power is absorbed by the arc. With respect to the rf energy absorbed in the breakdown, the behavior of SW structures is very different from TW structures [7]. In TW structures during most breakdowns, a large fraction of the incident energy is absorbed by the breakdown. In SW structures during most breakdowns, most of the rf power is reflected from the structure. Not all breakdowns in the SW structures have this character, some breakdowns do not produce large reflection.

\section{Simulations}

3D and 2D Particle-In-Cell simulations of the breakdown dynamics give a possible explanation of the breakdown development [7]. The simulation was based on a model of a "plasma spot" [8]. We assume that at some moment after a structure is filled with rf energy a plasma spot appears on the structure surface. The physics of the event that triggers the plasma spot in rf structures remains unclear.

The main results of these simulations for TW structures and waveguides are:

1. The major energy exchange between incident rf fields and particles comes from the interaction of the rf electric fields with electrons (not with ions). Electrons cross the gap in the waveguide or accelerating structure cell in a short time (about an rf period).

2. The electron current must be several kiloamps to significantly effect the rf power transmission. Space 
charge-limited emission of electrons without ions cannot produce and sustain the required current densities.

3. Ion currents of 10-100 A are needed to allow sufficient electron currents to disrupt transmitted power. The space charge field of the ions compensates the electron space charge field. This compensation allows the generation of kiloamps of electron current. The time constant for the drop off of transmitted power is $10-20 \mathrm{~ns}$ and is related mostly to the process of filling the waveguide gap or accelerating structure cell with copper ions.

4. A significant portion (50-80\%) of the emitted electrons and ions returns to the emitting spot and the surrounding area. We note that in the simulations a relatively large spot size (from $2 \mathrm{~mm}^{2}$ ) was used. The smallest spot size is determined by the minimal mesh size of the PIC code.

5. Up to $50 \%$ of the input power can be absorbed by the ion-electron beam in the waveguide and up to $60 \%$ in TW accelerating structure.

A theoretical model of the breakdown limit $[9,10]$ that uses the results of these simulations was able to explain the results of experiments with waveguides of different materials. The model assumes that the breakdown limit is set by melting of metal surface by arc electrons.

\section{RF Conditioning and Breakdown Limit}

$\mathrm{RF}$ conditioning is an important part of the rf breakdown study, since the breakdown limits and steady-state breakdown rates could not be reached without thousands of breakdowns at lower power and shorter pulse width.

The breakdown rate and the gradient limit for TW structures, SW structures and waveguides behave similarly with change of input rf parameters:

1. After-conditioning, breakdown limit is reproducible for structures of similar geometries. Typical variation of final gradient for the same structure material, geometry and breakdown rate is within 10-20\%. The number of breakdowns required to reach the limiting gradient varies greatly (sometimes by factors of 10) with the preparation of the structure surface. The source of this difference is not understood, and is being intensively studied. At the same time the limiting gradient for TW structures depends weakly on the surface preparation.

2. The breakdown rate grows almost exponentially with the power [5, 4, 11]. On a linear scale, it appears like the breakdown rate has a threshold.

3. At each stage of processing, the breakdown rate increases with increasing power and pulse width. The behaviour of the "constant-breakdown rate curve" usually could be fitted with function $P t^{\alpha}$. Here $P$ is the input power, $t$ is the pulse length. For low breakdown rates (far from the breakdown limit) $\alpha \sim 0.3$ [11]. Some experimental data obtained with TW structures suggest that at high breakdown rates (or near the breakdown limit) the $\alpha \sim 0.5$.

4. When a TW structure or waveguide is run with power and pulse length close to the limiting gradient, its breakdown behaviour significantly changes. "Breakdown chains" appear: after an initial breakdown, several more breakdowns cluster close to each other in location and time. In many cases secondary breakdowns follow after the initial breakdown at much lower power levels and pulse length, during the post breakdown procedure of ramping up of power and pulse length.

5. TW structures with higher shunt impedance that require less power to get to the same gradient have reached higher gradients than the structures with lower shunt impedance. The power at limiting gradient was about $90 \mathrm{MW}$ (400 ns pulse length) and it is roughly independent of structure type. For example, the high shunt impedance structure T53VG3MC has reached $90 \mathrm{MV} / \mathrm{m}$, and the the low shunt impedance H90VG3 have reached only $65 \mathrm{MV} / \mathrm{m}$ at similar breakdown rates of $<0.1 /$ hour and similar power of about $90 \mathrm{MW}$ [4].

6. The amplitude of the dark current emitted from the structures and waveguides follows a FowlerNordheim dependence on the surface electric field when fitted with field amplification factor $\beta$. For $\mathrm{X}$ band TW structures $\beta=30-40$. This value of $\beta$ is similar and reproducible for different TW accelerating structures.

7. The metal surface of the structure is obviously modified during rf processing in a way that it could sustain gradients higher than the gradients at start of rf processing. The physics of this process is still unclear. Observable parameters such as surface roughness are significantly worse (with craters and metal droplets) after processing compared to carefully prepared surfaces before processing.

The breakdown behaviour and the processing behaviour of TW structures and low-magnetic-field waveguides are similar. RF power at the breakdown limit is also similar ( 90 MW at $400 \mathrm{~ns}$ ). But peak amplitudes of surface electric fields are different. The surface field at the breakdown limit for waveguides is significantly lower than in accelerating structures (for the same pulse width). For example, at $400 \mathrm{~ns}$ pulse length, low-magnetic-field waveguide had a surface gradient of about $80 \mathrm{MV} / \mathrm{m}$ [6] while highest surface field in the TW53VG3MC TW structure [4] was about $200 \mathrm{MV} / \mathrm{m}$.

There are qualitative changes in breakdown behaviour of TW structures and waveguides near the gradient limit, such 
as concentration of breakdown locations and damage at the beginning of the structure and appearance of "breakdown chains". This change in breakdown behaviour suggests that there exists some kind of threshold combination of $\mathrm{rf}$ power and pulse length (reproducible from structure to structure) that sharply increases damage induced by breakdowns. We conjecture that this sets the gradient limit; increased damage prevents the structure from conditioning to higher gradients. A similar conjecture is used in [10].

\section{MOTIVATION}

Since the gradient limit for structures of the same geometry is weakly dependent on the initial condition of the structure it is possible that this limit is determined by the cell shape and the properties of the metal.

To study the effect of cell shape on rf breakdown behavior in a TW structure is difficult because the geometry of the cells varies along its length and rf power decays toward the end of the structure. Here we consider $11.424 \mathrm{GHz}$, near-constant-gradient TW structures developed for the NLC [3].

In order to study the effect of cell shape alone, we will perform experiments with single cell $T W$ structures. The idea is to build a structure that in only one cell mimics the fields near the input of a full-scale TW structure and has high electric and magnetic fields only in that cell, not in matching elements or couplers that transform the $\mathrm{TE}_{10}$ mode of rectangular waveguide into the "accelerating" circular $\mathrm{TM}_{01}$ mode. Another feature of the setup is the couplers, or mode launchers, which can be connected to the single cell structure with rf-compatible circular waveguide vacuum flanges. The flanges allow re-use of the same mode launchers for different structures, significantly reducing the cost of the experiments and allowing fast turn-around between tests of different structures.

We emphasize the difference between single cell $T W$ structures and earlier experiments with single cell structures [12]; the power available for breakdown and the field configuration in the single TW cell are similar to the power and fields at the beginning of the full TW accelerating structure. This was not the case in earlier experiments, which were done mostly with standing wave structures.

To study the difference between the breakdown behavior in TW structures and SW structures and the effect of SW cell shape on breakdown, we will also perform single cell $S W$ experiments. These will employ one of the mode launchers to feed rf power into a SW structure. Fields in the middle cell of the SW structure are similar to fields of a large-aperture SW structure already tested at high power (structure SW20a565 [5]). Fields in the other two cells are designed to be at most half that of the middle cell fields, so breakdowns will likely occur in the middle cell.

We emphasize that shapes and field distributions in both single cell TW and single cell SW structures are identical to shapes and fields of full-length structures. We speculate that this similarity will allow us to predict the behavior of practical structures from the tests. We note another advantage of the single cell structures: small geometry allows better diagnostics of breakdown events and makes possible detailed 3D simulation of breakdown processes observed in the experiments.

\section{SETUP}

The TW single cell setup consists of a TW structure in between two mode launchers. The SW single cell setup consists of one mode launcher and a SW structure. The mode launchers and standing wave structures have vacuum viewports to detect visible light and high frequency rf signals generated by breakdown events.

\section{Mode Launcher}

The mode launcher transforms the $\mathrm{TE}_{10}$ mode of WR90 rectangular waveguide into the $\mathrm{TM}_{01}$ mode in 0.900 inch diameter circular waveguide. It is designed as a single unit with rectangular and circular rf vacuum flanges and a vacuum viewport. A cutaway view of the mode launcher is shown in Fig. 1. Power in the WR90 input is split

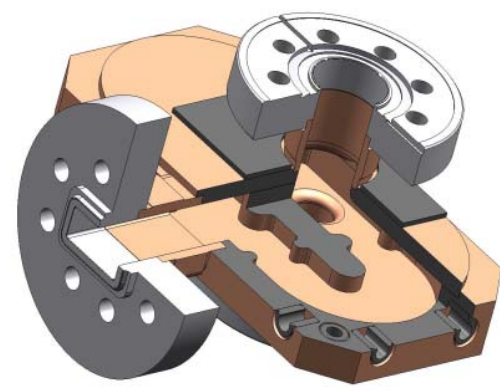

Figure 1: Cutaway view of the mode launcher.

with a matched $\mathrm{T}$, then symmetrically fed into the perpendicular circular waveguide. In the circular waveguide at $11.424 \mathrm{GHz}$, only two modes are supported: $\mathrm{TE}_{11}$ and $\mathrm{TM}_{01}$. With symmetric feeding, the $\mathrm{TE}_{11}$ mode is suppressed, and even with imperfect feeding symmetry it is poorly coupled to the rectangular $\mathrm{TE}_{10}$ mode (due to the field orientation). With only $\mathrm{TM}_{01}$ excited, a simple matching element in WR90 - a thick inductive iris - completes this launcher. Results of cold test measurement for the mode launchers are presented in [13]. Four mode launchers were built and successfully cold tested. Although the mode launcher itself has not been tested at high power, nine couplers for TW structures based on the design have been successfully used in the high-gradient structure program at SLAC [13].

\section{Travelling Wave Structures}

In the single cell $\mathrm{TW}$ setup, fields of the $\mathrm{TM}_{01}$ mode are first generated by the mode launcher, then transformed 
by a matching cell into fields of a periodic structure travelling wave (in one cell), then transformed through another matching cell back to the $\mathrm{TM}_{01}$ waveguide mode, to be extracted by the second mode launcher. The dimensions of the single cell are chosen to be the same as an initial cell of a TW structure known as T53VG3. This is a disk-loaded waveguide with initial group velocity of $3 \%$ the speed of light and $120^{\circ}$ phase advance per cell. The iris radius for the cell is $3.88 \mathrm{~mm}$, the iris thickness $1.66 \mathrm{~mm}$, the cell radius $10.641 \mathrm{~mm}$, and the cell length $8.747 \mathrm{~mm}$.

To create the travelling wave in one cell, we first match the 0.9 inch circular waveguide into a semi-infinite disk loaded waveguide using one matching cell. The matching process is described in detail in [13]. Then we make a structure with only one cell between two matching cells. Fields in this single cell structure are shown in Fig. 2. To

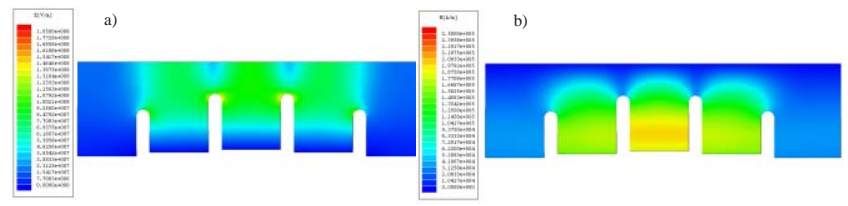

Figure 2: Amplitude of electric (a) and magnetic (b) fields in single cell TW structure for $40 \mathrm{MW}$ of input power.

illustrate the properties of the structure we show calculated reflection of the $\mathrm{TM}_{01}$ mode from a structure made of the same matching cells flanking one, four, and ten cells in Fig. 3. This graph clearly shows that the match at the

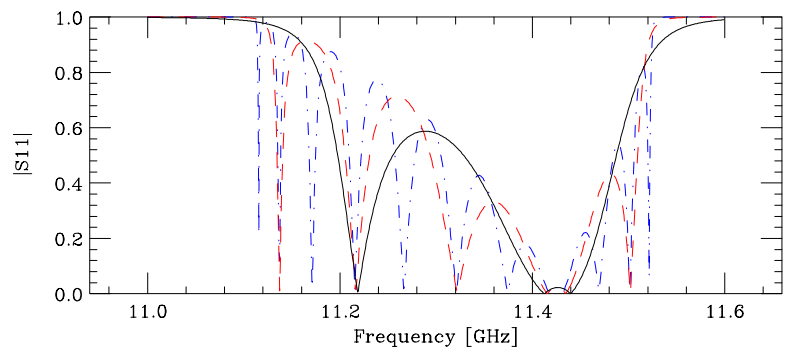

Figure 3: Reflection from a setup with different numbers of cells sandwiched between two matching cells: single cell (solid curve), 4 cells (dashed), and 10 cells (dot-dashed).

$11.424 \mathrm{GHz}$ working frequency is independent of the number of cells, i.e. that the matching cell creates fields with space harmonic content close to that of the travelling wave in periodic disk-loaded waveguide.

A total of five TW structures were built for the test, including one structure that has iris tips made of molybdenum. All of them were successfully cold tested. In Fig. 4, we show one of the TW structures with attached mode launchers during measurement of the field profile (beadpull test).

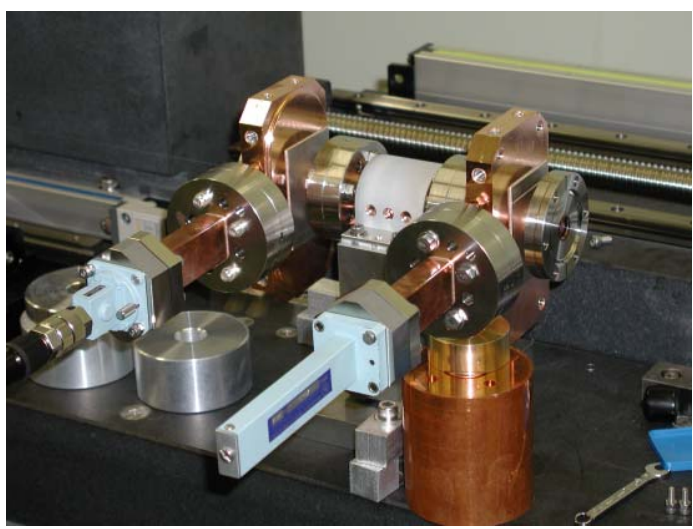

Figure 4: Single cell TW structure installed for bead-pull test.

\section{Standing Wave Structures}

For the single cell SW setup we used the dimensions of the NLC prototype SW structure SW20a565, with $\pi$ phase advance per cell [5]. The SW20a565 cell dimensions are: cell radius $1.150 \mathrm{~mm}$, cell length $11.312 \mathrm{~mm}$, iris radius $5.65 \mathrm{~mm}$, iris thickness $4.6 \mathrm{~mm}$, major-axis of the elliptical iris tip $3.4 \mathrm{~mm}$. In the single-cell SW structure, the cell radius was slightly modified to achieve the desired field profile.

We added two cells to the structure to create $\pi$-like fields in the test cell: a matching cell connected to circular waveguide and an end cell with a vacuum viewport. The geometries of these two cells were chosen such that the structure is matched at the working frequency (critical coupling) and the maximum fields in these cells are half as large as the fields in the test cell.

The structure was designed with the 2D finite element code SLANS [14]. During the matching, four parameters were adjusted: the radii of all cells and the iris radius of the matching cell. The cell radii determine the field profile in the structure, and the iris of the matching cell determines the coupling of the structure to the circular waveguide. Afterward, the matching was verified with the commercial electrodynamic code HFSS [15]. The field distribution in a copper single cell SW structure is shown in Fig. 5. We note that the same design procedure could be applied to structures with more than one cell for future study of the effect of the number of cell on breakdown behavior. We

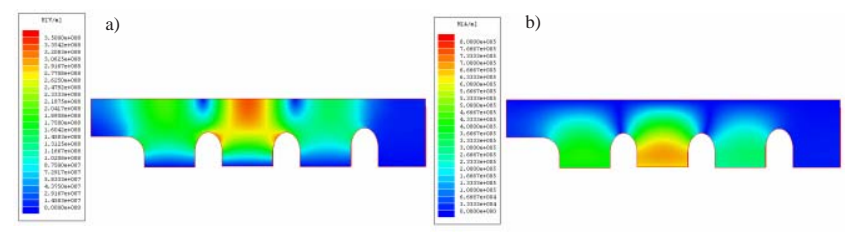

Figure 5: Amplitude of electric (a) and magnetic (b) fields in single cell copper SW structure for $10 \mathrm{MW}$ of input power. The structure fed from the right; on the left, there is an opening with vacuum viewport. 
made two designs, one for testing of copper structure and another one for molybdenum structure. Since the resistivity of molybdenum is higher than that of copper, we changed the radii of all cells and radius of the matching iris of the molybdenum structure in order to maintain the field profile and the critical coupling.

To date, a total of two copper SW structures have been built and successfully cold tested.

\section{PROPOSED EXPERIMENTS}

The experiment will be conducted at the SLAC Klystron Test Area using a SLAC solenoid focused XL4 klystron. Initially, we have $40 \mathrm{MW}$ and $1.5 \mu s$ available for the test. We may increase the power at the expense of the shortening the pulse length using a pulse compressor. To contain dark current and the breakdown induced radiation from the cavities, we use a box with four inch thick lead walls.

\section{Different Materials}

With copper TW and SW structures, we plan to study the effect of cell shape, circuit (SW vs. TW), and surface preparation methods on breakdown behavior. In addition, we have built a TW structure with iris tips made of molybdenum, and are building a TW and a SW structure made of solid molybdenum. We are going to compare breakdown limits and the conditioning behavior of these structures and the copper structures.

The structure shapes (for both SW and TW structures) and rf parameters, such as power and pulse width, for the these experiments will be close to those of practical structures. The importance of the choice of rf parameters was shown in experiments with a CERN molybdenum TW structures, where the short-pulse breakdown limit was higher than for copper. A similar structure operated at longpulse length did not reach the copper breakdown limit [16].

\section{Surface Processing}

We are going to study the effect of different surface processing on conditioning and breakdown behavior. We will use copper TW and SW structures for this experiment. We are going to compare standard SLAC/KEK processing of high gradient structures, high pressure water rinsing, and light etching of the assembled structure. The standard procedure includes etching of the structure cells only before bonding. All the structures will be assembled in a clean room and vacuum baked at $600^{\circ} \mathrm{C}$ for several days.

\section{REFERENCES}

[1] ILC TRC Report, SLAC-0606, 2003.

[2] C. Adolphsen et al., "High Gradient Performance of Prototype NLC/GLC X-Band Accelerator Structures," ROAC004, this conference.

[3] J. Wang and T. Higo, "Accelerator structure development for NLC / GLC,” ICFA Beam Dyn. Newslett. 32, 27 (2003).
[4] C. Adolphsen, "Normal Conducting RF Structure Test Facilities and Results," ROPC006, PAC03, Portland, Oregon, May 12-16, 2003.

[5] V. A. Dolgashev et al., "Status of X-band Standing Wave Structure Studies at SLAC," TPAB031, PAC03, Portland, Oregon, May 12-16, 2003.

[6] V. A. Dolgashev, S. G. Tantawi, "RF Breakdown in X-band Waveguides,” TUPLE098, EPAC'02, 3-7 June, 2002, Paris, France, pp. 2139-2141.

[7] V. A. Dolgashev, S. G. Tantawi, "Simulations of Currents in X-band accelerator structures using 2D and 3D particlein-cell code," FPAH057, PAC 2001, June 18-22, Chicago, Illinois. pp. 3807-3809.

[8] P. B. Wilson, "A Plasma Model for RF Breakdown in Accelerator Structures," LINAC 2000, Monterey, CA, August, 2000.

[9] P. B. Wilson, SLAC-PUB-9953, PAC03, Portland, Oregon, 12-16 May 2003.

[10] P. B. Wilson, SLAC-PUB-11086, LINAC 2004, Lubeck, Germany, 16-20 Aug 2004.

[11] C. Adolphsen, "Advances in Normal Conducting Accelerator Technology from the X-Band Linear Collider Program," TOPE002, this conference.

[12] J. W. Wang, "RF Properties of Periodic Accelerating Structures for Linear Colliders," SLAC-Report-339, Ph.D. Dissertation, Stanford University, 1989.

[13] C. Nantista, S. Tantawi and V. A. Dolgashev, "Low-field accelerator structure couplers and design techniques," Phys. Rev. ST Accel. Beams 7, 072001 (2004), 7 pages.

[14] D. G. Myakishev et al., "An interactive code SLANS for evaluation of RF-cavities and accelerator structures," Proceedings of IEEE PAC01, 1991, San Francisco, Ca, pp.3002-3004.

[15] http://www.ansoft.com/products/hf/hfss/

[16] W. Wuensch et al., "A High-Power Test of an XBand Molybdenum-Iris Structure," THP34, LINAC 2004, Lubeck, Germany, 16-20 Aug 2004. 